\title{
Bases de mármol para un techo efímero. Preguntas sobre lo que permanece en el Museo Nacional de Bellas Artes de Buenos Aires
}

\section{Articulo de reflexión}

\section{Julia Isidori}

Conicet, UNRN, UNTreF, Argentina juli91_2006@hotmail.com

Recibido: 21 de enero de 2019

Aprobado: 13 de marzo de 2019

Cómo citar este artículo: Isidori, Julia (2020). Bases de mármol para un techo efímero. Preguntas sobre lo que permanece en el Museo Nacional de Bellas Artes de Buenos Aires. Calle 14: revista de investigación en el campo del arte 15(27). pp. 108-125. DOI: https://doi. org/10.14483/21450706.15415

Agradezco a Carolina, bibliotecaria del Museo Nacional de Bellas Artes, y al Departamento de Documentación y Archivo. El presente trabajo fue una investigación en el marco de la Maestría en Curaduría y el Doctorado en Teoría Comparada de las Artes de la Universidad Nacional Tres de Febrero, Buenos Aires.

\section{(a) (1)}

https://creativecommons.org/licenses/by/4.0/deed.es 


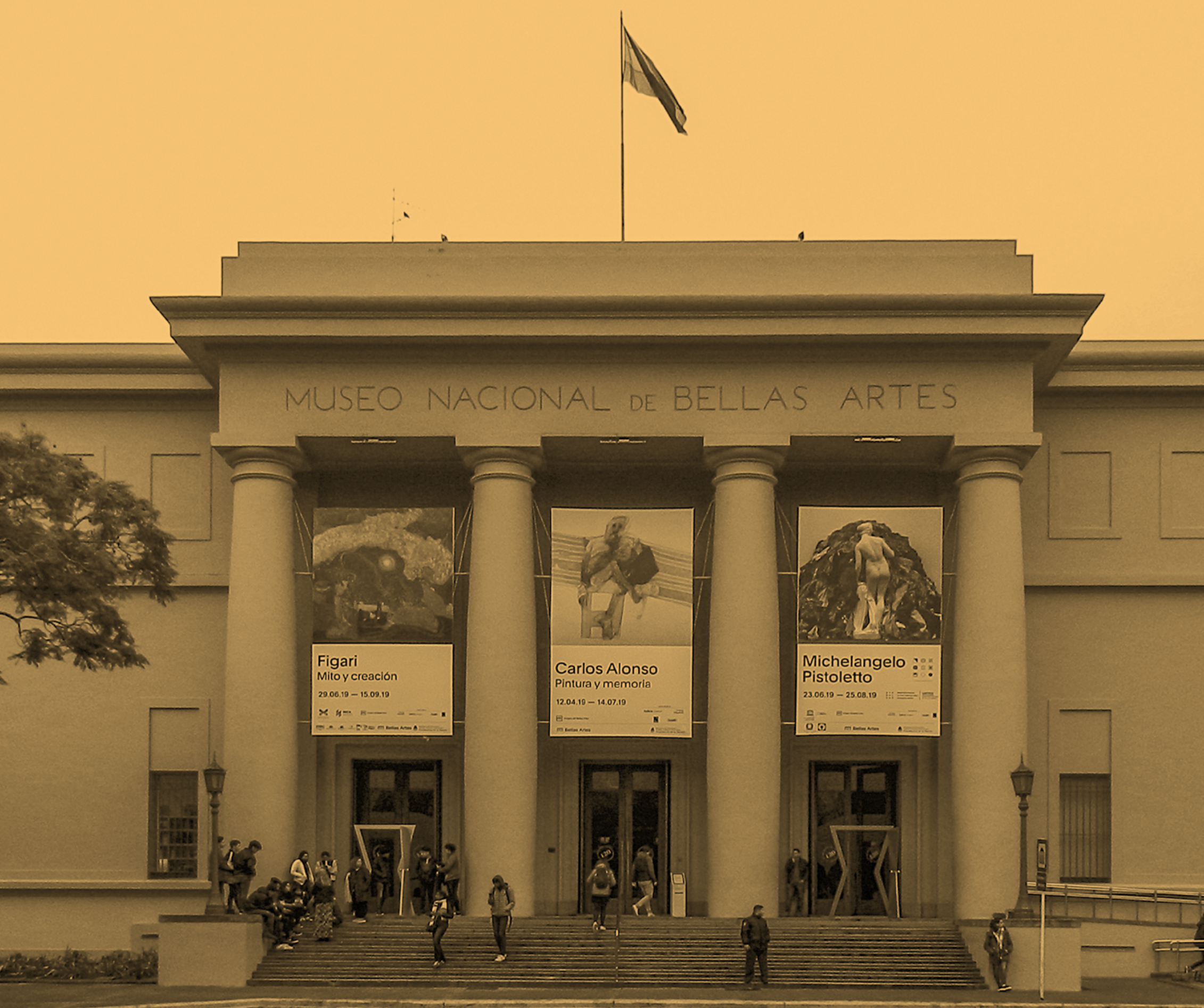


Bases de mármol para un techo efímero. Preguntas sobre lo que permanece en el Museo Nacional

de Bellas Artes de Buenos Aires

\title{
Resumen
}

En este ensayo nos preguntamos cómo el Museo Nacional de Bellas Artes de Buenos Aires (MNBA) ha venido adquiriendo rasgos modernizadores, eurocéntricos y elitistas desde su fundación en 1896 al día de hoy. Para ello, nos concentramos en las colecciones permanentes -especialmente en la sala 3 de la planta baja - y analizamos la paradoja de su carácter privado en el seno de una institución pública. Mediante el relevamiento de catálogos de los últimos 50 años, análisis de la sala misma y consultas al departamento de Documentación y Archivo, notamos la coerción que ejercen los donantes de colecciones privadas sobre el museo, no solo en el relato curatorial de lo exhibido, sino también sobre lo que se decide no mostrar. En este panorama, nos preguntamos qué posibilidades tiene la institución de tomar decisiones curatoriales y de formación de públicos desde una perspectiva de Estado.

\section{Palabras clave}

Colección de arte; Estado; museo

Marble Bases for an Ephemeral Roof. Questions About What Remains at the National Museum of Fine Arts in Buenos Aires

\begin{abstract}
In this essay we wonder how the National Museum of Fine Arts of Buenos Aires (MNBA) has been acquiring modernizing, Eurocentric and elitist features since its founding in 1896 to today. To do this, we focus on the permanent collections-especially in room 3 on the ground floor-and analyze the paradox of its private nature within a public institution. Through the survey of catalogs from the last 50 years, an analysis of the room itself and consultations with the Department of Documentation and Archive, we note the coercion that donors of private collections exert on the museum, not only in the curatorial narrative of the exhibits, but also on what they decide not to show. In this scenario, we wonder what possibilities the institution really has of making curatorial decisions and training its audiences from a public sector perspective.
\end{abstract}

\section{Keywords}

Art collection; State; museum

\section{Bases en marbre pour un toit éphémère. Questions sur ce qui survit dans le Musée National des} Beaux-Arts de Buenos Aires

\section{Résumé}

Dans cet essai, nous nous demandons comment le Musée national des beaux-arts de Buenos Aires (MNBA) a acquis des traits modernistes, eurocentriques et élitistes depuis sa fondation en 1896 jusqu'à nos jours. Pour ce faire, nous nous concentrons sur les collections permanentes - en particulier dans la salle 3 du rez-de-chaussée - et analysons le paradoxe de sa nature privée au sein d'une institution publique. À travers l'enquête sur les catalogues des 50 dernières années, une analyse de la salle elle-même et des consultations avec le Département de documentation et d'archives, nous notons la contrainte exercée sur le musée par les donateurs de collections privées, et cela pas seulement dans la narrative des expositions, mais aussi dans ce qu'ils décident de ne pas montrer. Dans ce scénario, nous nous demandons quelles sont les possibilités réelles de l'institution de prendre des décisions de conservation et de former son public d'un point de vue étatique. 


\section{Mots clés}

Collection d'art ; Etat ; musée

Bases de mármore para um telhado efêmero. Perguntas sobre o que resta no Museu Nacional de Belas Artes de Buenos Aires

\section{Resumo}

Neste ensaio, nos perguntamos como o Museu Nacional de Belas Artes de Buenos Aires (MNBA) vem adquirindo características modernizantes, eurocêntricas e elitistas desde sua fundação em 1896 até hoje. Para isso, nos concentramos nas coleções permanentes - especialmente na sala 3, no térreo - e analisamos o paradoxo de sua natureza privada dentro de uma instituição pública. Através da pesquisa de catálogos dos últimos 50 anos, uma análise da própria sala e consultas com o Departamento de Documentação e Arquivo, notamos a coerção que os doadores de coleções particulares exercem sobre o museu, não apenas na narrativa curatorial das exposições, mas também sobre o que eles decidem não mostrar. Nesse cenário, nos perguntamos quais são as possibilidades que a instituição realmente tem de tomar decisões curatoriais e treinar suas audiências a partir de uma perspectiva de Estado.

\section{Palavras-chave}

Coleção de arte; Estado; museu

\section{Basekuna mármol kumbrirapa uiarasina tapuikuna imasatak ka}

\section{Maillallachiska}

Kai ensaiopi tapurrinakunchi imasatak museo nacional Bellas Artes de Buenos Aires mansa (MNBA) samunaku surkiaska kauai musukuna eurocéntricos i elitistakuna kallariska uramanda 1896 uatamanda kunauramanda. Chipak iuiarinchimi tandachiskakunapi uku kimsapi uralado- i kauanakuchimi chi paradojak suma cuidaspa chuchupe institución publicape. Mediante chi relevamientoua katatogokunaua kai pichka chunga uatapi,agllanakui chi ukupi tapunakui Departamento documentacionta i iakachiskape, kauanakunchi chi coerción rurankuna karagkuna chi colección privadokuna muscopi manaparlollapi curatorialpi kauachiskapi manakagpik niskamanda mana kauachiskamanda. Kai panoramapi tapurinakunchi imasatak ka chi institución apingapa desicionkuna curatorialkuna i uiñachii publikupi prespectiva estadomanda.

\section{Rimangapa Ministidukuna}

Tandachiska arte; estado; museo 


\section{Vuelo de pájaro. Mirada panorámica sobre el Museo}

En este trabajo se analizarán algunos aspectos de la exposición permanente de la Colección Hirsch que ocupa la sala N. 03 del Museo Nacional de Bellas Artes de la ciudad de Buenos Aires. La misma será estudiada en relación al periplo histórico de la institución, al circuito espacial que su arquitectura propone y a las injerencias de estos dos factores sobre la construcción de sentido que se habilitan en las y los espectadores que la visitan, en el marco de una institución pública nacional latinoamericana.

La intención de analizar una sala particular sin desentendernos del espacio mayor que la contiene responde por un lado al propósito de situar el objeto de estudio y por otro, al recaudo de no correr el riesgo de resignar el análisis de la sala al mero estudio de las obras que exhibe. Consideramos que, fuera de estas, los elementos propios de la colección reunida en un recinto particular -el espacio, las disposiciones en él, el criterio curatorial- son inevitablemente parte del resto del museo. ${ }^{1}$ Además, cabe mencionar que las salas como las conocemos hoy datan a penas de 1977, fecha antes de la cual el museo pasó por siete mudanzas y reformas municipales. Por ello, antes de abocarnos especialmente a la sala Hirsch, consignaremos brevemente algunos ejes sobre los que observamos se estructura el Museo Nacional de Bellas Artes: eurocentrismo, modernidad y elitismo.

El primero ha signado sin tregua el carácter de la institución desde sus inicios, afectando desde las propias obras que conforman el acervo, estén en bodega o expuestas, hasta los referentes culturales que se vincularon a la dirección del Museo. Para comenzar por las obras, observemos que ya en las primeras adquisiciones a fines del siglo XIX, las piezas europeas constituían un 91\% del patrimonio. A partir de un estudio sobre el Primer Catálogo publicado en 1896 por el MNBA, constatamos

1 Para citar un ejemplo, si eligiéramos, por caso, la sala N.․ 8, que exhibe grabados y pinturas de Francisco de Goya, y no atendiéramos a los criterios que la exceden, perderíamos de vista nada menos que la razón que la puso allí: una especie de segunda opción o "premio consuelo" de Brest —según María José Herrera-, quien hubiese preferido los inadquiribles Picasso. Ante la imposibilidad económica del museo para adquirir estas obras del y exhibirlas como epítome de la modernidad e ideal de culminación del arte para su director en ese momento, Brest acepta las donaciones - primero para ser exhibidas y luego para ser incluidas en el acervode Horacio Acevedo (Herrera, 2009, p. 18).

\begin{tabular}{|c|c|c|c|}
\hline Sala & $\begin{array}{l}\text { Autor/a (o n. }{ }^{\circ} \text { de } \\
\text { inventario por sala) }\end{array}$ & Lugar de pertenencia & $\begin{array}{l}\text { Cantidad } \\
\text { de obras }\end{array}$ \\
\hline \multirow[t]{17}{*}{1} & Claudio Lastra & Arg. Fran. & 1 \\
\hline & Richter Edouard & Escuela Francesa & 1 \\
\hline & Charton Ernest & Escuela Francesa & 1 \\
\hline & Laar Pieter Van & Escuela Holandesa & 2 \\
\hline & Manzoni Ignacio & Escuela Italiana & 12 \\
\hline & Blanes Juan M & Uruguay & 1 \\
\hline & Vera M & Argentina & 1 \\
\hline & Hamond & & 2 \\
\hline & El Canaletto & Italia & 2 \\
\hline & Teniers David & Escuela Flamenca & 1 \\
\hline & Paris Alfred & Arg. Fran. & 1 \\
\hline & Abbate Nicolo Dell' & Italia & 1 \\
\hline & Agrelo Mariano & Arg. Italia & 1 \\
\hline & $\begin{array}{l}\text { Pueyrredon Maximiliano } \\
\text { Paz }\end{array}$ & Arg. Fran. España & 1 \\
\hline & 29,31 & Esuela Francesa & 2 \\
\hline & Boneo Martin L & Escuela Italiana & 2 \\
\hline & Dresco Arturo & Italia & 1 \\
\hline \multirow[t]{7}{*}{2} & 1,3 & Escuela Italiana & 2 \\
\hline & De Martino, Edoardo & Escuela Italiana & 1 \\
\hline & 3 & Inglaterra & 1 \\
\hline & Manzoni, Ignacio & Escuela Italiana & 2 \\
\hline & Blanchin Emile Henri & Escuela Francesa & 1 \\
\hline & Boneo Martin L & Argentina & 2 \\
\hline & Agrelo Mariano & Arg. Italia & 1 \\
\hline \multirow[t]{21}{*}{3} & $\begin{array}{l}\text { Puvis de Chavannes } \\
\text { Pierre }\end{array}$ & Escuela Francesa & 1 \\
\hline & Manzoni Ignacio & Escuela Italiana & 10 \\
\hline & Luminais Evariste Vital & Escuela Francesa & 1 \\
\hline & 4,22 & Escuela Flamenca & 2 \\
\hline & 5 & Escuela Italiana & 1 \\
\hline & Cerezo Mateo & Escuela Española & 1 \\
\hline & Monvoisin Raymond & Escuela Francesa & 1 \\
\hline & Stadler & Escuela Alemana & 1 \\
\hline & 11 & Escuela Española & 1 \\
\hline & Ward James & Escuela Inglesa & 1 \\
\hline & Van Leemputten & Escuela Flamenca & 2 \\
\hline & Doré Gustave & Escuela Francesa & 1 \\
\hline & Escuela de Van Loo Carle & Francia & 1 \\
\hline & Schgoer F & Escuela Inglesa & 1 \\
\hline & Vleughels Nicolás & Escuela Francesa & 1 \\
\hline & A.A. 28 & Escuela Francesa & 1 \\
\hline & Ballerini Augusto & Arg. Italia & 1 \\
\hline & Sterner Albert & Norteamérica & 1 \\
\hline & Montenard Frederic & Escuela Francesa & 1 \\
\hline & 32,32 & Jesuita & 2 \\
\hline & Mendilaharzu Graciano & Argentina & 1 \\
\hline
\end{tabular}




\begin{tabular}{|l|l|l|l|}
\hline Sala & $\begin{array}{l}\text { Autor/a (o n.o de } \\
\text { inventario por sala) }\end{array}$ & Lugar de pertenencia & $\begin{array}{l}\text { Cantidad } \\
\text { de obras }\end{array}$ \\
\hline 3 & Veiver Van der & Escuela Flamenca & 1 \\
\cline { 2 - 4 } & Lastra Claudio & Arg. Italia & 1 \\
\hline 37 & Escuela Bolognesa & 1 \\
\hline & Boilly Luis Leopold & Escuela Italiana & 1 \\
\hline
\end{tabular}

\begin{tabular}{|l|l|l|l|}
\hline 4 & Escuela Española & 1 \\
\hline Boneo Martín L & Argentina & 1 \\
\hline Escuela de Guido Reni & Italia & 1 \\
\hline Schleh Anna & Escuela Alemana & 1 \\
\hline Fra Sebastiano & Escuela Italiana & 1 \\
\hline Piazzetta Giovanni & Italiana & 1 \\
\hline Escuela Benozzo Gozzolli & Italia & 1 \\
\hline Bellangé Jean L & Escuela Francesa & 1 \\
\hline Lastra Claudio & Argentina & 1 \\
\hline Agujari Giuseppe & Italia & 1 \\
\hline Giordano Luca & Escuela Italiana & 1 \\
\hline Escuela de Guardi & Escuela Italiana & 2 \\
\hline Francesco & & \\
\hline Manzoni Ignacio & Escuela Italiana & 6 \\
\hline Morono Giovanni Battista & Escuela Italiana & 1 \\
\hline 15 & Escuela Francesa & 1 \\
\hline Escuela de Nicolás & Escuela Italiana & 1 \\
\hline Poussin & & \\
\hline 18 & Escuela Flamenca & 1 \\
\hline Curtois Jacques & Escuela Francesa & 1 \\
\hline Agujari Tito & Escuela Italiana & 1 \\
\hline Correa Morales Lucio & Argentina & 1 \\
\hline Agrelo Mariano & Argentina & 1 \\
\hline De Martino Edoardo & Escuela Italiana & 1 \\
\hline Escuela de Pannini & Escuela Italiana & 1 \\
\hline Wissot de Warville Félix & Escuela Francesa & 1 \\
\hline Giúdici Reynaldo & Argentina & 1 \\
\hline \hline & Escuela Alemana & 2 \\
\hline Boos Filipp & Escuela Italiana & 1 \\
\hline Escuela Francesa & 1 \\
\hline & & \\
\hline Bontana & & \\
\hline
\end{tabular}

\begin{tabular}{|l|l|l|l|}
\hline 5 & Erespi Daniele & Escuela Italiana & 1 \\
\cline { 2 - 3 } & Arellano Juan de & Escuela Española & 1 \\
\hline Mancini Antoni & Escuela Italiana & 1 \\
\hline Azeglio Mássimo & Escuela Italiana & 1 \\
\hline Ryng Pierre de & Escuela Flamenca & 1 \\
\hline Peluso F & Escuela Italiana & 1 \\
\hline Le Roy Pierre Francoise & Escuela Flamenca & 1 \\
\hline 8 & Jesuita & 1 \\
\hline Lami Luis E & Escuela Francesa & 1 \\
\hline Le Beaud Edmond & Francia & 1 \\
\hline Hernández Daniel & Perú & 1 \\
\hline 11 & Escuela Italiana & 1 \\
\hline Manzonni Ignacio & Escuela Italiana & 6 \\
\hline
\end{tabular}

\begin{tabular}{|l|l|l|l|}
\hline Sala & $\begin{array}{l}\text { Autor/a (o n. } \\
\text { inventario por sala) }\end{array}$ & Lugar de pertenencia & $\begin{array}{l}\text { Cantidad } \\
\text { de obras }\end{array}$ \\
\hline & Rousseau Théodore & Escuela Francesa & 2 \\
\hline Bruyn Barthelemy & Escuela Alemana & 1 \\
\hline Rigaud Hyacinthe & Escuela Francesa & 1 \\
\hline 19 & Escuela Italiana & 1 \\
\hline Ponte Jacopo da & Escuela Italiana & 1 \\
\hline Utrecht Adrien van & Escuela Flamenca & 1 \\
\hline Roll Alfred & Escuela Francesa & 1 \\
\hline Pueyrredon Prilidiano Paz & Arg. Fran. España & 1 \\
\hline Wilkie David & Escuela Inglesa & 1 \\
\hline Ribot Theodule & Escuela Francesa & 1 \\
\hline Ostade Adrien van & Escuela Holandesa & 1 \\
\hline Palleja José Miguel & Uruguay España & 1 \\
\hline Hosthorst Gerard Van & Escuela Holandesa & 1 \\
\hline Esc de Guido Reni & Escuela Italiana & 1 \\
\hline Palamedes Antonio & Escuela Holandesa & 1 \\
\hline Sánchez Coello Alonso & Escuela Española & 1 \\
\hline Esc de T. Giovanni & Escuela Italiana & 1 \\
\hline Battista & & \\
\hline Charton Ernes & Escuela Francesa & 1 \\
\hline Rosa Salvatot & Escuela Italiana & 1 \\
\hline Cárcano Filipo & Escuela Italiana & 1 \\
\hline
\end{tabular}

Obras ignotas sin registro de Escuela o procedencia

\begin{tabular}{|l|l|l|}
\hline Sala & $\begin{array}{l}\text { Autor/a (o n.o de } \\
\text { inventario por sala) }\end{array}$ & $\begin{array}{l}\text { Cantidad } \\
\text { de obras }\end{array}$ \\
\hline 1 & 28 & 1 \\
\hline 30 & 34 & 1 \\
\hline 3 & 34 & 1 \\
\hline & 19 & 1 \\
\hline 5 & 39 & 1 \\
\hline
\end{tabular}

Gráfico 1. Obras que figuran en el Primer Catálogo del Museo Nacional de Bellas Artes, publicado en 1986. (Nota: Cuando figura más de un país en la columna de procedencia, el primero corresponde a la nacionalidad, y los siguientes a la formación artística.) 


\begin{tabular}{|l|l|l|l|l|l|l|l|l|l|l|}
\hline América colonial y poscolonial & América precolombina & Europa & Asia & Oceanía & Sin datos \\
\hline 6704 & 3551 & 6776 & 3051 & 18 & 0 & 2650 & \\
\hline
\end{tabular}

Gráfico 2. Obras pertenecientes al patrimonio del MNBA, clasificadas cuantitativamente por lugar de procedencia. (Información obtenida del archivo actualizado del patrimonio, facilitado por la Lic. Paula Casajús, Jefa del área de Documentación y Registro del MNBA. Junio de 2018).

que, en ese año, después de dos donaciones de familias acaudaladas de Buenos Aires, 144 obras pertenecían a las escuelas francesa, italiana, flamenca, alemana, española, e inglesa, mientras que solo 14 eran originarias de nuestro continente (uno de un pintor norteamericano, uno de origen uruguayo, uno proveniente de Perú, tres de misiones jesuíticas y ocho de artistas argentinos, a su vez formados en Italia, España o Francia). Este patrimonio inaugural fue anunciado seis años antes de que el museo adquiriera por sí mismo obras según el criterio de su director, en ese entonces Eduardo Shiaffino, quien llevó a cabo la tarea en el viejo continente.

Por otra parte, trasladándonos a un tiempo más próximo, el Catálogo Razonado en dos Volúmenes, coordinado por Roberto Amigo y publicado por la institución en 2010, asegura un gran número dentro del inventario patrimonial de piezas de arte precolombino (Amigo, 2010, p. 17). Sin embargo, estas no están exhibidas. Comparar porcentajes de piezas provenientes de Europa y piezas latinoamericanas en el acervo permite dilucidar el nivel de detalle que se tiene sobre las primeras -tanto en patrimonio como en conocimiento y puesta en valor de las obras - en contrapunto con las originarias de este territorio, catalogadas bajo entradas más amplias justamente debido al número total de inventario. ${ }^{3}$ Este fenómeno se puede observar cotejándolo con la cronología de exposiciones ${ }^{4}$ desde la primera gestión, dirigida por Schiaffino desde 1896 hasta el año 1993. A partir de 1994, con el mandato de Jorge Glusberg, las exposiciones temporarias aumentan, dando protagonismo a artistas latinoamericanos, pero en carácter de

2 Se trata de las familias Guerrico y Rossi, que inmediatamente después del decreto publicado por Uriburu para fundar el Museo Nacional, se incluyeron a sí mismas en la tarea de constituir un acervo.

$3 \quad$ Nótese, por ejemplo, que en las exposiciones permanentes y temporarias de arte europeo está anunciado el recorte cronológico, disciplinar y geográfico limitado a países (por ejemplo, Pintura francesa del S. XIX, mientras que las de arte latinoamericano pecan de una generalidad causada por la escasez de piezas. Es por esto que se titula, por ejemplo, Arte precolombino.

$4 \quad$ Ver sección La historia en la página web del MNBA. Disponible en: https://www.bellasartes.gob.ar/museo/historia. Consultado en enero de 2018. invitación temporal y siempre a producciones del S. XX. Constatamos, entonces, que la inespecificidad latinoamericana sobre arte anterior al S. XVIII no se resuelve.

Si observamos el plano actual de exhibiciones permanentes, confirmaremos que en la planta baja hay un total de 21 salas destinadas a obras de origen europeo y solo tres que exhiben arte argentino, cabe aclarar que puesto en valor en relación a escuelas del viejo continente. En el tiempo que duró la presente investigación -abril a junio de 2018-, pudieron constatarse, de las obras efectivamente exhibidas en las salas permanentes del MNBA, 379 piezas de origen europeo, 177 latinoamericanas, de las cuales 65 se produjeron bajo la formación de escuelas europeas, y 12 asiáticas. $^{5}$ En palabras de María José Herrera, "el relato que este guion evidencia es el de relación de escuelas e influencias que posicionan al arte argentino en el contexto de la historia del arte occidental, es decir europeo. Las comparaciones no apuntan a denotar la especificidad de arte argentino sino la filiación de la producción local y su pertenencia a una cultura internacional. (Herrera, 2009 , p. 20). Es cierto que estos datos corresponden a la planta baja, y que si observamos los planos del $1^{\circ}$ y el $2^{\circ}$ piso descubriremos exposiciones de arte latinoamericano y argentino. Pero, en primer lugar, el primer piso está destinado en gran parte a las exhibiciones temporarias, y en segundo lugar, son las salas de la planta baja las que establecen el primer contacto con el/la visitante del museo, por lo que tienen inevitablemente un carácter programático de lo que vendrá. En este sentido, las exposiciones de arte europeo que ocupan el $78.16 \%$ de la superficie total, sientan el principio eurocéntrico que rige la lectura de todo lo exhibido (incluso resulta sumamente llamativa, en la página web del MNBA, el hecho de que la pestaña "obras no exhibidas" es prácticamente un catálogo de arte prehispánico, lo que da cuenta a primera vista de qué es lo que deliberadamente queda fuera de las exhibiciones).

$5 \quad$ No ahondamos en este trabajo en la inclusión de arte proveniente de otras geografías, como Asia, África u Oceanía. 


\section{Planta baja}

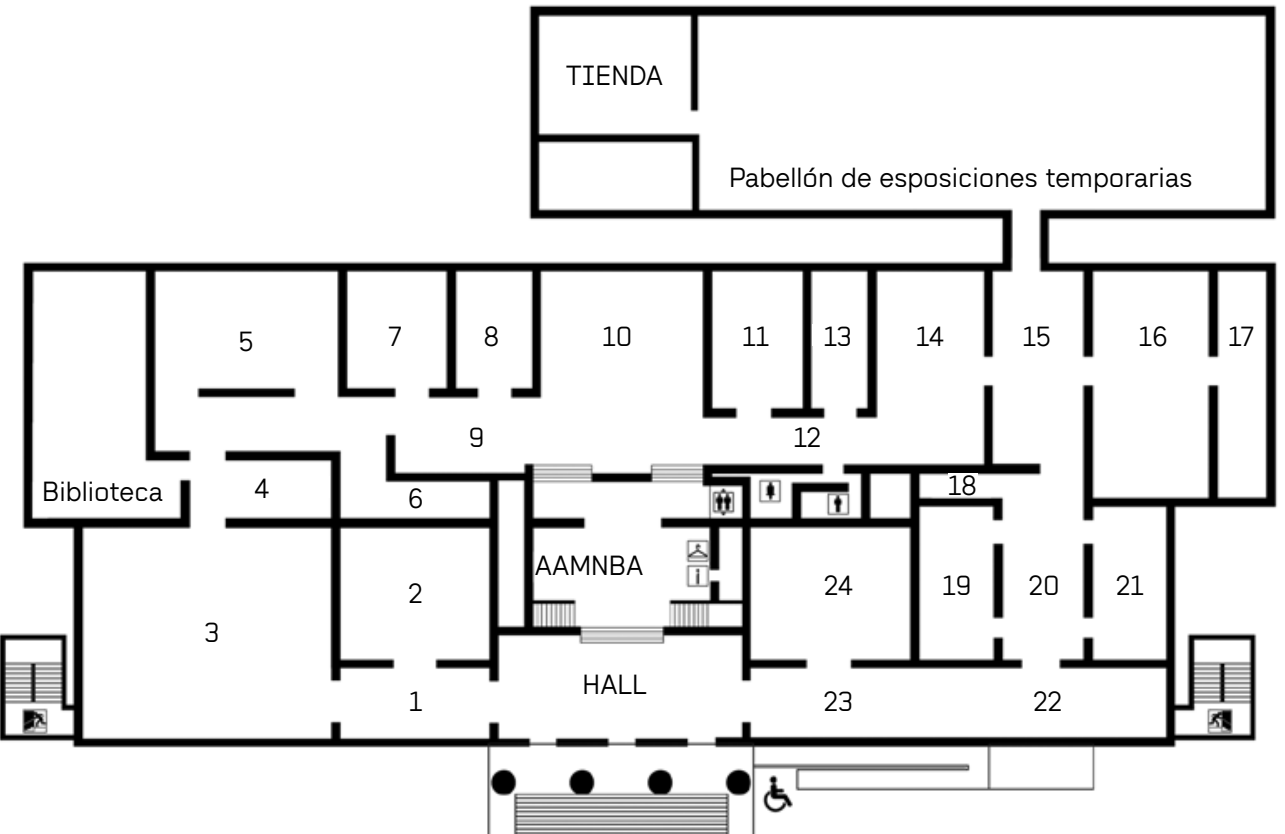

Av. del Libertador

1-2 Arte europeo, siglo XII al XVI

3 Colección Hirsch

4 Pintura holandesa y flamenca

5 Manierismo y barroco

6 Colección Emilio P. Furt

7 Pintura del siglo XVIII
Franciso de Goya

Pintura francesa, siglo XIX

Pintura y escultura francesa, siglo XIX

Arte italiano, siglo XIX

Arte europeo, siglo XIX

Pintura europea, siglo XIX
14 Impresionismo y postimpresionismo

15 Colección Santamarina

16-17 Colección Guerrico

18-19-20 Colección Santamarina

21 Sala temporaria

22-23-24 Arte argentino, siglo XIX

\section{Primer piso}

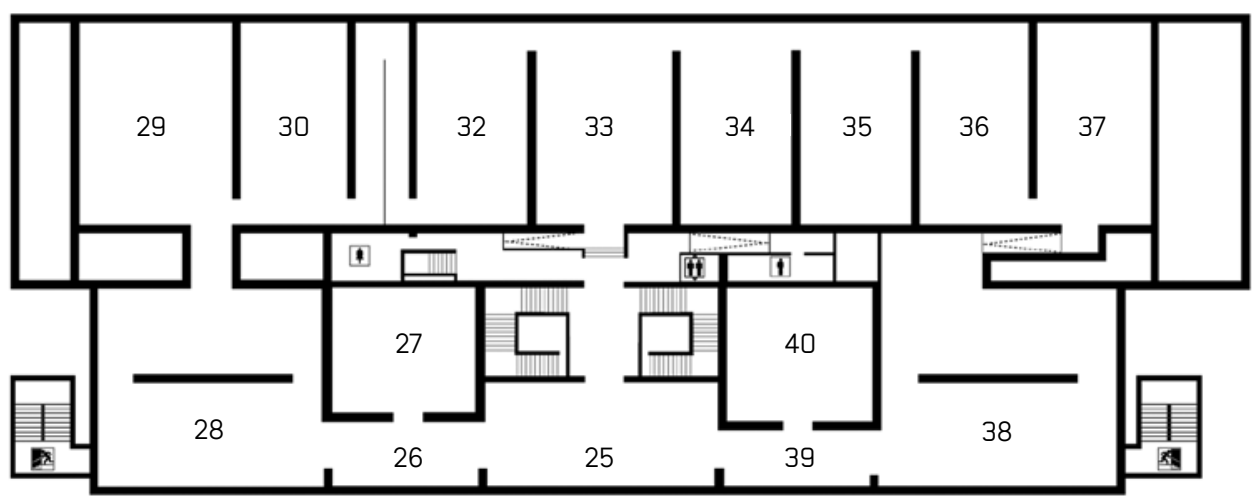

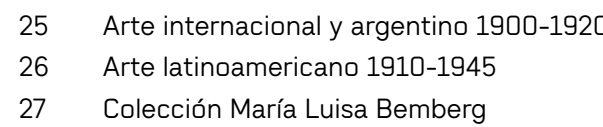

28-29-30 Arte internacional y argentino 1920-1945
32 Arte latinoamericano y argentino 1910-1945

33 Antonio Berni

34-35-36 Arte argentino 1945-1970

37 Arte internacional y argentino 1945-1970
38-39 Arte argentino 1960-1980

40 Arte argentino de los 80

\section{Segundo piso: exposiciones temporarias}


En resumidas cuentas, el museo fija la idea de que el arte americano-argentino no está delimitado por el territorio, sino por la historia de la conquista. La institución se desentiende, en sus primeros 50 años de formación, de las producciones americanas, y, cuando las incluye, fija una relativa data de génesis precisamente concordante con el inicio de la Nación, a principios del S. XIX. Nótese, por ejemplo, que la exposición La pintura y la escultura argentinas de este siglo remarca incluso desde su título el límite decimonónico, límite que no es tal para el resto de las colecciones del museo, como si de Europa pudieran mostrarse producciones incluso del S. XII, pero lo americano meritorio de ser exhibido datara recién del 1800. Justamente a partir de ese entonces el museo comenzó a entablar un vínculo con Europa por medio de sus estudiantes de arte a la manera de un discípulo aplicado, dispuesto a olvidar lo necesario y a aprender los conocimientos de sus maestros. Las y los artistas argentinos recorrían en su formación los pasos de las diversas escuelas europeas y según la tradición, los estudiantes más valorados eran premiados con un viaje a Europa como una suerte de culminación de los estudios con nada menos que "Ios verdaderos maestros".

Es así que observamos que la postura eurocéntrica del Museo no afecta solo su patrimonio: determina también, desde los inicios, la postura ideológica y los vínculos políticos que la dirección entablará con otras entidades en su carácter de representación nacional de la cultura en Argentina. Es sorprendente observar en un recorrido cronológico de las primeras décadas, como desde un principio Buenos Aires estableció vínculos permanentes con capitales de Europa, mientras que no se menciona un solo contacto con países de América que atravesaban un similar período de conformación nacional (El Museo Nacional de Bellas Artes de Chile se inauguró en 1880, 15 años antes que el MNBA de Argentina, el de Río de Janeiro en 1937, el de Paraguay en 1909, el de Montevideo en 1911, entre otros).

Consecuentemente se adivina sin problemas la posición que el país buscaba en el panorama mundial. En este sentido, es lógico el traslado en 1910 de la construcción de hierro y vidrio que en 1989 hubiera oficiado como Pabellón Argentino en la Feria Universal de París ${ }^{6}$ para ser inaugurado como sede del Museo (Herrera, 2010, p. 20). También es lógico que, en esta intención eurocén-

6 A favor de nuestra hipótesis cabe señalar que, en la exposición universal, este pabellón llevó a la muestra de las naciones dos obras de Rodin. trica de poner en valor la nación respaldándose en las urbes europeas y no en los territorios vecinos que estuvieran atravesando períodos de independencia respecto de Europa, la dirección del MNBA, representada en ese entonces por Carlos Zuberbühler, invitara a ser protagonistas de la Exposición del Centenario de la Revolución de mayo a países como Francia, Austria, Hungría, Estados Unidos e Italia (AAVV2007: 10).

Mediante una aproximación al acervo y a la historia fundacional de la institución hemos confirmado el carácter eurocéntrico que la misma adquirió desde sus comienzos. Un segundo eje que a nuestro parecer estructura al Museo Nacional de Bellas Artes es la modernidad, anunciada literalmente como meta a perseguir por varios de sus directores, entre los que podríamos mencionar a Eduardo Schiaffino, Atilio Chiáppori y, el más reciente, Romero Brest. Por cuestiones de extensión, y porque nuestro objeto de estudio es otro, no daremos ejemplos que den cuenta de las intenciones modernizantes del museo, pero sí quisiéramos detenernos en el siguiente punto: por muy universal que se nos haya instalado, "modernidad" es, desde el vamos, un concepto extranjero. Esto nos permite identificar cómo la institución nacional del arte se organizó siguiendo un criterio y cronologías que lo ubican inevitablemente en la periferia. En este sentido, el propósito mayor de la institución se presenta como algo imposible deencarnar, dejando a la Argentina como mera espectadora de la modernización ajena: los referentes son siempre externos, y los modelos de progreso están siempre fuera de las fronteras. Por más desalentador que esto parezca es una consecuencia inevitable, si los valores propios de una cultura autóctona no se ponen en valor independientemente de criterios extranjeros. ${ }^{7}$

Por último, el tercer eje que reconocemos como estructura del Museo Nacional de Bellas Artes es el elitismo. Qué entendemos por un "museo nacional" depende, grosso modo, de lo que entendamos por "lo nacional": si consideramos que lo nacional está representado sobre todo en los grupos populares, que representan la mayoría demográfica, o si por el contrario entendemos que la determinación de lo nacional delimita las

7 Resulta interesante observar que, durante la gestión de Teresa Parodi como Ministra de Cultura, la edición de la Guía anual del museo empezaba por primera vez con una imagen americana (AAVV, 2015, p. 25). Sin embargo, en los catálogos posteriores, las obras reproducidas en las primeras páginas que marcan el terreno sobre los criterios de valor que adopta el museo, volvieron a ser europeas, por lo general renacentistas. Véanse los catálogos de los años 2006, 2007, 2010 para mencionar publicaciones recientes. 


\begin{tabular}{|c|c|c|c|c|c|c|c|}
\hline Sala & A & Af/E & E & As & Don. & Leg. & Adq. \\
\hline 1 y 2 & & & 24 & & 21 & 2 & 1 \\
\hline 3 & & & 15 & & 15 & & \\
\hline 4 & & & 5 & & 1 & 2 & 3 \\
\hline 5 & & & 17 & & 11 & 2 & 4 \\
\hline 6 & & & 8 & & 5 & & 3 \\
\hline 7 & & & 9 & & 6 & 1 & 2 \\
\hline 8 & & & 17 & & 15 & 1 & 1 \\
\hline 9 & & & 5 & & 2 & & 3 \\
\hline $10-\mathrm{Nov}$ & & & 21 & & 20 & & 1 \\
\hline 12 & & & 10 & & 6 & & 4 \\
\hline 13 & & & 8 & 12 & 3 & 1 & 4 \\
\hline 14 & & & 15 & & 6 & 1 & 8 \\
\hline 15 & 23 & & $110^{*}$ & & $454^{*}$ & & \\
\hline $16-17$ & & & 11 & & 23 & & \\
\hline 18 & & & 4 & & 4 & & \\
\hline 19 & & & 14 & & 13 & & 1 \\
\hline 20 & & 10 & 3 & & 4 & 1 & 8 \\
\hline 21 & & & 7 & & 2 & 2 & 3 \\
\hline 22 & 9 & 7 & & & 12 & & 4 \\
\hline 23 & 9 & & & & 9 & & \\
\hline 24 & & 9 & & & & & 9 \\
\hline Esc. & 3 & & & & 2 & & 1 \\
\hline 25 & & 3 & 8 & & 4 & 1 & 6 \\
\hline 26 & 4 & 6 & & & 7 & 1 & 3 \\
\hline 27 & 14 & 13 & & & 27 & & \\
\hline 28 & 4 & 7 & 11 & & 5 & 4 & 12 \\
\hline 29 & 6 & 4 & 17 & & 19 & 2 & 7 \\
\hline 30 & 16 & 2 & 2 & & 9 & 1 & 10 \\
\hline 34 & 1 & 3 & 13 & & 5 & & 12 \\
\hline 35 & 18 & & & & 14 & & 4 \\
\hline 36 & 5 & 1 & 3 & & 6 & & 3 \\
\hline TOTAL & 112 & 65 & 379 & 12 & 733 & 22 & 117 \\
\hline
\end{tabular}

aspiraciones de un grupo reducido que se considera en la potestad de educar; si creemos que la identidad está construida desde adentro, o si en cambio depende de lo que el exterior designe en nosotros. Hemos de considerar, de todas maneras, que estas son opciones entre muchas de las posibles, y que, a su vez, no pueden ser modificadas de forma abrupta por cada nueva gestión, o al menos no lo han sido. En su lugar, a lo largo de su historia el museo fue adicionando modos de considerar lo nacional, pero no ha podido desprenderse de las bases elitistas que lo estructuraron hacia finales de 1800 y principios del S. XX. La causa es que gran parte del patrimonio proviene de donaciones, es decir, no de la determinación de un grupo directivo sobre lo que consideran apropiado mostrar en un museo nacional, sino de lo que las familias más pudientes del país deciden regalar. Pero no nos detendremos acá en este eje, porque lo retomaremos a propósito de la Colección Hirsch, que lo ejemplifica a la perfección. Solo mencionaremos al respecto que, durante la investigación, 733 de las obras exhibidas en las salas permanentes provenían de donaciones, 22 de legados, y tan solo 117 de adquisiciones del museo.

En este panorama -eurocéntrico, modernizador, y elitista - se inscribe la sala a la que nos avocaremos en el siguiente apartado.

\section{Enfoque de presa. Vista de águila sobre la Colección Hirsch}

Previo a la determinación de una sala de la planta baja para analizar, en la tarea de recabar información sobre la exposición Hirsch —que me presentaba inquietudes

Gráfico 3. Cantidad de obras exhibidas actualmente (otoño-2018) en las salas permanentes del museo, clasificadas por lugar de procedencia (E: Europa, A: América, As: Asia) y modo de ingreso al patrimonio (Don: Donación, Leg: Legado, Adq: Adquisición).Nota: En la sala N. 15, correspondiente a la donación Guerrico, no se contabilizan en tabla, en la clasificación por origen, las 323 piezas exhibidas en vitrina, por no contar con mayor información que su número de inventario. Sí se tienen en cuenta en la casilla de Donación.

de tipo curatorial-, el personal de la Biblioteca me explica que obtendría mayor información del personal de seguridad que custodia sus obras que de los archivos bibliográficos. Razón de ello es el carácter privado de dicho espacio, que depende no de la institución nacional, sino de la familia de donantes. Esta decide qué se exhibe, bajo qué criterios curatoriales, las características espaciales de la sala y los elementos indicadores de recorrido. Incluso es la responsable del sueldo del empleado de seguridad, quien, al preguntarle por la persona o el equipo de profesionales responsable de la curaduría y cuándo fue la última vez que la sala fue modificada, me responde: "Acá nadie puede tocar nada. Ni el director del museo se puede meter acá." Su defensiva respuesta, casi un alarde de poder, terminó de esclarecer que analizar una de las salas que menos dependen del museo es una oportunidad para estudiar 


\begin{tabular}{|c|c|c|c|c|}
\hline Autor/a & Obra & Detalles & Procedencia & N.․ de inventario \\
\hline Philippe Alexius de Laszlo de Lombos & Retrato de Doña Elizabeth G. de Hirsch & $\begin{array}{l}1933 \text { Óleos tela, } \\
100 \times 74 \mathrm{~cm}\end{array}$ & Hungría/ G Bretaña & 8641 \\
\hline Philippe Alexius de Laszlo de Lombos & Retrato de Don Alfredo Hirsch & $\begin{array}{l}1933 \text { Óleos tela, } \\
99 \times 74 \mathrm{~cm}\end{array}$ & Hungría/ G Bretaña & 8633 \\
\hline Rembrandt van Rijn y Taller & Retrato de mujer joven & $\begin{array}{l}1634 \text { Óleos tela, } \\
63 \times 56 \mathrm{~cm}\end{array}$ & Holanda & 8622 \\
\hline $\begin{array}{l}\text { Flinck Govaert (discípulo de } \\
\text { Rembrandt) }\end{array}$ & Retrato de joven con sombrero con plumas & $\begin{array}{l}\text { S. XVII Óleos tela, } \\
85 \times 73 \mathrm{~cm}\end{array}$ & Holanda & 8625 \\
\hline Licino Bernardino & Retrato de un magistrado & $\begin{array}{l}1520 \text { Óleos tela, } \\
73 \times 62 \mathrm{~cm}\end{array}$ & Italia & 8628 \\
\hline Aert de Gelder & $\begin{array}{l}\text { Ester y Mardoqueo escribiendo la primera } \\
\text { carta del Purim }\end{array}$ & $\begin{array}{l}1645-1727 \text { Óleos tela, } \\
111 \times 132 \mathrm{~cm}\end{array}$ & Holanda & 8643 \\
\hline $\begin{array}{l}\text { Talleres de Francoise de La Planche } \\
\text { y Marc de Comans }\end{array}$ & $\begin{array}{l}\text { El capitán a caballo (Serie la Hisotira de } \\
\text { Artemisa }\end{array}$ & $\begin{array}{l}1617 \text { Lana, seda e } \\
\text { hilos de oro y plata, } \\
441 \times 449 \mathrm{~cm}\end{array}$ & Bélgica & 8634 \\
\hline Peter Paul Rubens & Alegoría de la fortuna y la virtud & $\begin{array}{l}\text { S. XVII Óleos tela, } \\
36 \times 42 \mathrm{~cm}\end{array}$ & Alemania/Bélgica & 8629 \\
\hline Anónimo (copia de Peter Paul Rubens) & El rapto de las Sabinas & $\begin{array}{l}\text { S. XVI-XVII Óleos } \\
\text { tela, } 57 \times 89 \mathrm{~cm}\end{array}$ & Escuela Flamenca & 8630 \\
\hline Anónimo (Taller de Frans Hals) & Retrato de un caballero & $\begin{array}{l}\text { S. XVII Óleos tela, } \\
36 \times 42 \mathrm{~cm}\end{array}$ & Escuela Holandesa & 8626 \\
\hline Adriaen Van de Velde & Playa de Scheveningen & $\begin{array}{l}1636-1672 \text { Óleos tabla, } \\
22 \times 27 \mathrm{~cm}\end{array}$ & Holanda & 8631 \\
\hline Paulus Moreelse & Retrato de Caballero & $\begin{array}{l}1636-1672 \text { Óleos tabla, } \\
72 \times 56 \mathrm{~cm}\end{array}$ & Holanda & 8644 \\
\hline $\begin{array}{l}\text { Anónimo (según el estilo de Giovanni } \\
\text { di Bartolomeo) }\end{array}$ & Madonna con el niño & $\begin{array}{l}\text { S. XIX Piedra y madera } \\
80 \times 38 \mathrm{~cm}\end{array}$ & Italia & 8645 \\
\hline Lucas Cranach el viejo & Crucifixión de Cristo & $\begin{array}{l}\text { S. XVI Óleos tabla, } \\
85 \times 62 \mathrm{~cm}\end{array}$ & Alemania & 8632 \\
\hline Adriaen Isenbrant & Santos y donantes & $\begin{array}{l}\text { 1490-1551 Óleo } \\
\text { y témperas tabla, } \\
73 \times 23 \mathrm{~cm}\end{array}$ & Bélgica & $\begin{array}{l}8627 / 02 / 01- \\
8627 / 02 / 02\end{array}$ \\
\hline Jacob Cornelisz van Oostzanen & El nacimiento de la virgen María & $\begin{array}{l}1470-1533 \text { Óleos tabla, } \\
129 \times 75 \mathrm{~cm}\end{array}$ & Holanda & 8624 \\
\hline Herri Met de Bles & La Última aparición de Cristo a sus discípulos & $\begin{array}{l}\text { 1510-1150 Óleos } \\
\text { tabla, } 77 \text { x } 97 \mathrm{~cm}\end{array}$ & Bélgica & 8623 \\
\hline Anónimo & Armario & $\begin{array}{l}\text { S. XVII Madera, } \\
212 \times 170 \times 71 \mathrm{~cm}\end{array}$ & Holanda & 8646 \\
\hline Anónimo & San Antonio Abad & $\begin{array}{l}\text { S. XV Madera con } \\
\text { restos de policromía, } \\
81 \times 15 \times 27 \mathrm{~cm}\end{array}$ & Alemania & 8636 \\
\hline Anónimo & Nessus y Dejanira & $\begin{array}{l}\text { S. XVII Bronce, } \\
41 \times 20 \times 30 \mathrm{~cm}\end{array}$ & Italia & 8637 \\
\hline Anónimo & Hércules y Cacus & $\begin{array}{l}\text { S. XVII Bronce, } \\
42 \times 15 \times 15 \mathrm{~cm}\end{array}$ & Italia & 8638 \\
\hline Anónimo & Ninfa tocando una trompeta & $\begin{array}{l}\text { S. XVII Bronce, } \\
24 \times 7 \times 7 \mathrm{~cm}\end{array}$ & Escuela Flamenca & 8640 \\
\hline Anónimo & Dios Pan & $\begin{array}{l}\text { S. XVIII Bronce, } \\
30 \times 9 \times 11 \mathrm{~cm}\end{array}$ & Italia & 8639 \\
\hline - & Piedad & & & \\
\hline Anónimo & Cabeza de Séptimo Severo & $\begin{array}{l}\text { S. XVI Mármol, } \\
35 \times 25 \times 28 \mathrm{~cm}\end{array}$ & Italia & 8635 \\
\hline Anónimo & Neptuno con tridente & $\begin{array}{l}\text { S. XVII Bronce, } \\
76 \times 22 \times 37 \mathrm{~cm}\end{array}$ & Italia & 8647 \\
\hline
\end{tabular}

Gráfico 4. Obras exhibidas en la actual Sala 3 del MNBA, pertenecientes a la Colección Hirsch, por orden de aparición en sentido horario desde el intgreso, primero en las paredes y luego las del centro, siguiendo una espiral y según los datos en sala. 
la naturaleza del mismo. Una suerte de mirada de Troya desde el interior del caballo.

Tal como lo aseguró el responsable de custodia, la sala, que forma parte del recorrido del museo desde el año $1983{ }^{8}$ es una suerte de reino soberano dentro del territorio nacional. El museo puede tener sus reglas, sus criterios estéticos y críticos de exhibición, pero la sala está eximida de acatarlos. ¿Por qué forma parte, entonces, de una institución de carácter público? ¿Por qué goza de los privilegios estatales ${ }^{9}$ y de difusión de un museo nacional?

Simultáneamente a la pretensión de independencia respecto del resto de la institución, forma parte de su recorrido y recibe sus mismos visitantes, por lo que podríamos preguntar, ante la coexistencia de relatos diferentes ¿quién se subsume, esta colección o el resto del museo? Temo que el segundo, porque ve sus intenciones curatoriales -independientemente de que sean constelacionarias, lineales o de otros esquemasinterrumpidas por puntos inamovibles, imposibles de ser modificados.

Observemos, por ejemplo, el plano actual del museo (figura 1) teniendo en mente el criterio cronológico propuesto por el circuito de la planta baja: ingresando por el ala este (hacia la izquierda desde la entrada), los visitantes comienza el recorrido ante piezas de origen europeo que datan desde el S. XI hasta el XIV, en su mayoría esculturas y pinturas de la imaginería católica, seguidas por obras producidas desde esa fecha hasta el S. XVI (salas 1 y 2 ) advirtiendo a primera vista el ordenamiento cronológico. Las paredes son lisas y están pintadas de color oscuro, hay luces puntuales para iluminar cada pieza de forma direccionada, rayas de grises bajos en el suelo indicando distancia obligatoria entre las obras y los espectadores, y el texto de sala ploteado en la pared da cuenta del criterio con que las mismas fueron seleccionadas y ordenadas. La sala 4 repetirá estos patrones, junto con la 5ㅇ, 6ㅇ, 70 y 8 y las que van desde la 11 hasta la 24 . Observamos que hay tres salas

8 El espacio que hoy ocupa era el utilizado para la Biblioteca del Museo, de carácter público desde 1942. Con el ingreso de la colección Hirsch, esta fue desplazada a un anexo que le resta visibilidad.

9 Me refiero desde la superficie en m2 para exhibir su colección, hasta la comodidad de que aquellos que la visiten puedan usar los baños del resto del museo. En otras palabras, la familia Hirsch hace alarde de su patrimonio - cuyo valor se incrementa con la exhibición ante tamaña cantidad de espectadores - sin las obligaciones administrativas de sostener una galería (según el personal de entrada, el MNBA está recibiendo alrededor de 1.500 visitas por día, que llegan a triplicar su número en fines de semana), que escapan a estas regularidades: La9 y la 10 solo se diferencian en sus paredes blancas y las luces generales, aunque el resto de los elementos se mantienen.

Sin embargo, la sala Hirsch, N. .3 , presenta alteridades a todos los criterios del resto del museo: Las paredes claras tienen entrantes que enmarcan las piezas y les dan mayor o menor relevancia visual; las luces son generales, y las marcas de distancia estipulada con los espectadores se elevan desde el suelo en barandas doradas. Los textos de sala están grabados en placas de acrílico sostenidas por pies dorados, y sus leyendas no comparten ni el tipo de escritura de los ploteos de las salas restantes ni su apariencia (tipografía, color, etc.). Las obras, aunque son todas de origen europeo, no siguen un orden cronológico ni geográfico por países entre ellas, y tampoco continúan el relato lineal de las salas que las preceden y de las que siguen.

En este sentido, la colección Hirsch es una suerte de galería aparte que no da cuenta del criterio bajo el que las obras están reunidas y mucho menos organizadas en el espacio. Lo que sí anticipa en su ingreso es la propiedad de las mismas, en la parte superior de la puerta de entrada (Imagen 2) y en una placa de mármol, empotrada en la pared, enmarcada en bajorrelieve y grabada (Imagen 3) con la siguiente leyenda:

\author{
IN MEMORIAM \\ de Don Alfredo Hirsch \\ (1872-1956) \\ y su esposa Doña Elizabeth Gottschalk de Hirsch \\ (1888-1965) \\ Esta sala y las obras de arte que en ella se exhiben \\ han sido donadas al Museo de Bellas Artes \\ de la República Argentina, por sus herederos: \\ Mario Hirsch \\ Sarah Saavedra de Hirsch \\ Claudia Caravallo de Quentin \\ Octavio A. Caraballo \\ Buenos Aires, 1984.
}

Exactamente debajo de la misma hay un soporte de acrílico transparente que, aunque hace tres meses está vacío, está destinado a sostener los textos de sala que pueden retirar los visitantes.

En cuanto a la exposición, las obras (Gráfico 4) van desde un mueble de madera del S. XII hasta una tabla religiosa de segunda mitad del S. XIX, pasando por bustos renacentistas, paisajes flamencos y hasta los retratos del Sr. y la Sra. Hirsch, que datan de principios 


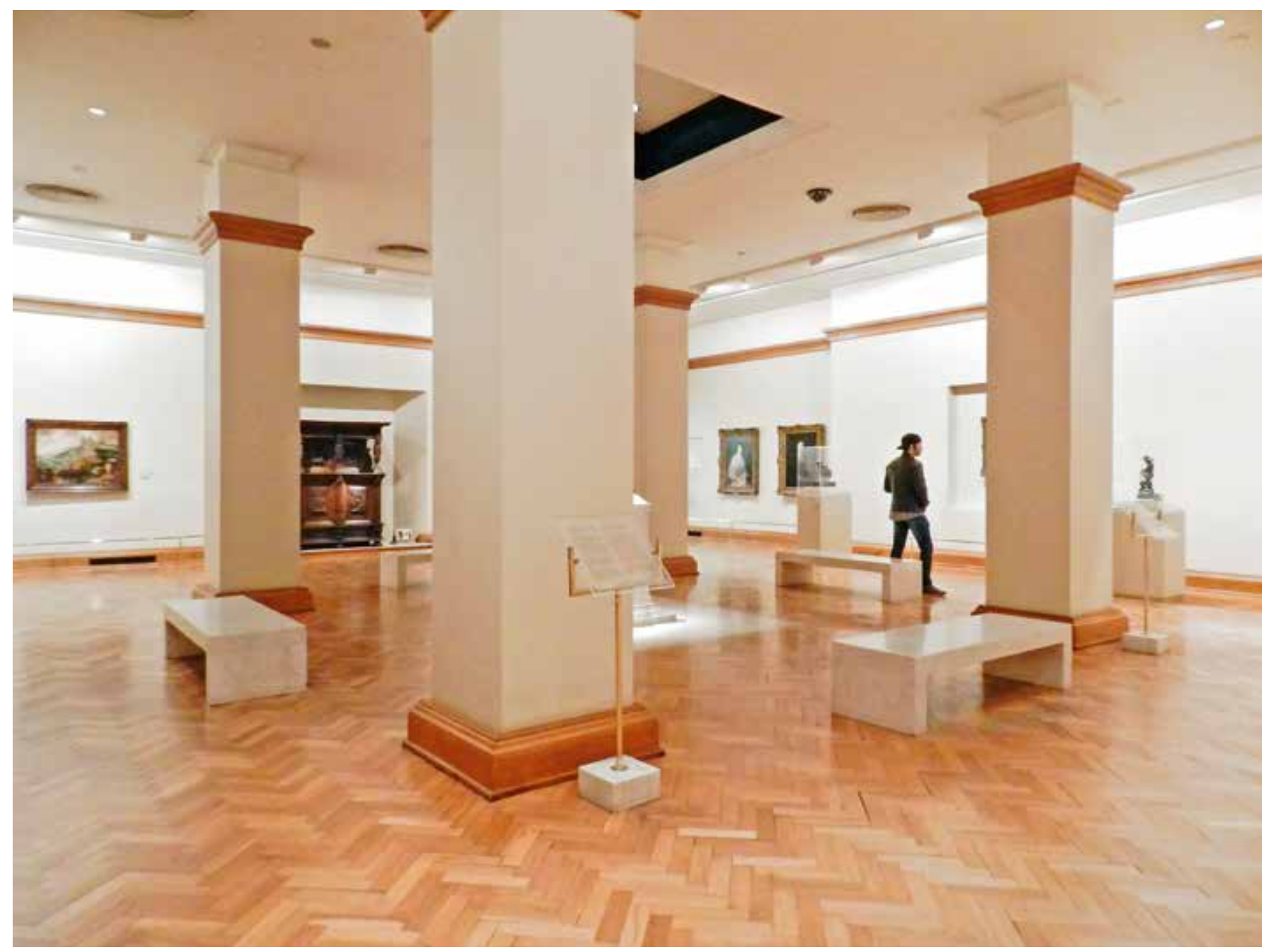

Imagen 2. Vista del ingreso e interior de la sala 3 del MNBA, Colección Hirsch.

de siglo XX. Como hemos dicho, dispuestas sin obedecer a un orden cronológico o territorial, ni mucho menos temático. Acerca de los dos retratos, es necesario mencionar que comparten la ubicación en el ingreso de la sala, al lado de la placa recordatoria, haciendo notar el dominio sobre la colección y su organización, que no pertenecen en sí mismos a un relato cronológico determinado sino inaugurado por ellos. El retrato es un género predominante en la colección Hirsch, que podría sugerir un sistema de valores alrededor del ensalzamiento de personas particulares.

En cuanto al espacio general (Imagen 2), los detalles arquitectónicos y objetos espaciales dan cuenta de la no pertenencia de la sala al resto del museo. Asimismo, el tipo de materiales - parqué de diseño complejo (esto está en resto del museo), barandas doradas, cuatro columnas de $75 \mathrm{~cm}$ de lado en medio de la sala, molduras de madera en zócalo, cielorraso y columnas, como también molduras en las bases de las esculturas, ocupando mayor dimensión que las obras en sí- interrumpen la vista de las obras y desvían la atención a la distinción del edificio. Los bancos son de diseño minimalista pero su corporeidad también suma un elemento más que compite con los objetos de arte, o que más bien habilita una construcción de sentido particular, asociado a la alcurnia nacional y a la colección de obras como exhibición de riqueza y decoración. Lo mismo ocurre con las paredes, que, si bien son claras, justamente es por esta cualidad que acentúan los detalles decorativos arquitectónicos ya mencionados, así como las entrantes en los muros sugieren jerarquía entre las obras.

La mayoría de estos elementos fueron evitados en las otras salas del MNBA, lo que sugiere la intervención de profesionales en su curaduría. Sin embargo, la sala Hirsch está notablemente diseñada, pero siguiendo criterios de decoración de interiores y mobiliario. No hay mayor recaudo en el modo en que las obras se enfrentan a los espectadores -incluso dejando a un lado la selección 


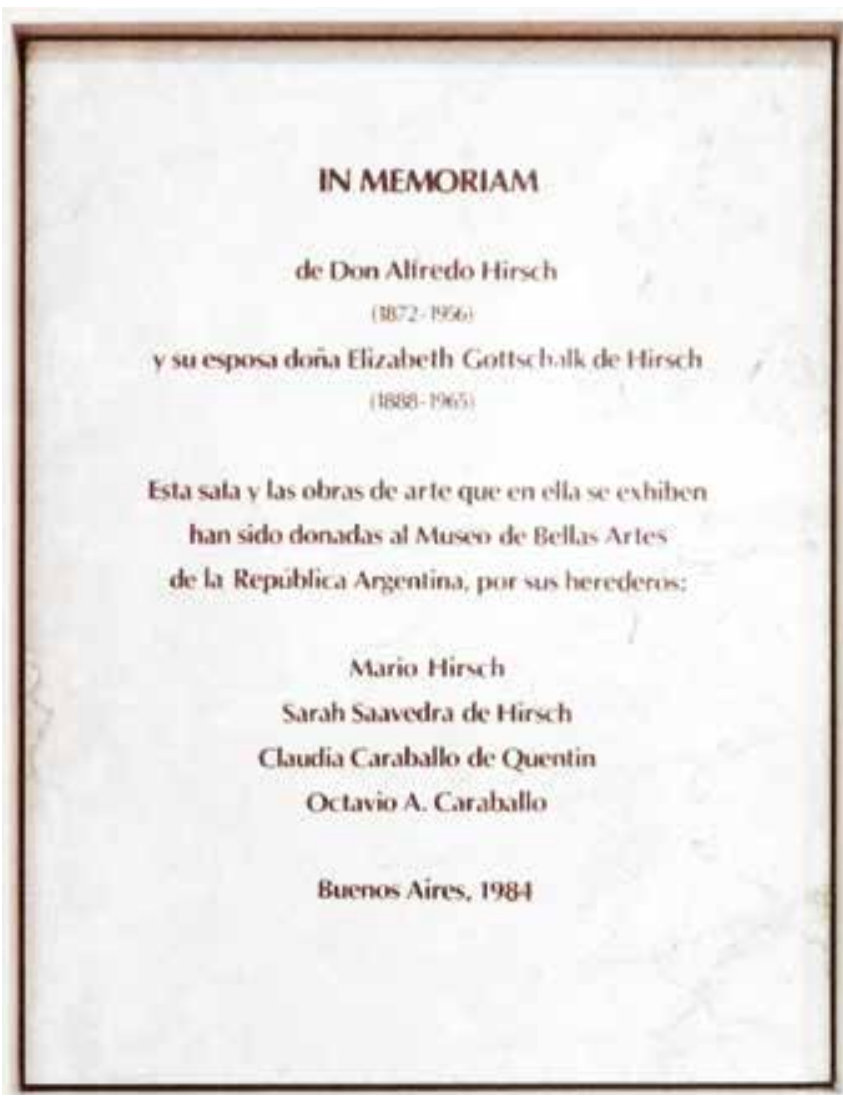

Imagen 3. Placa conmemorativa ubicada en la pared sur de la sala.

total-, como puede comprobarse en la mirada individual de las piezas. Nótese, por ejemplo, que el armario del S. XVII (ubicado en la pared sur) está escoltado por dos objetos contemporáneos de funcionamiento mecánico cuya función no puede adivinarse. ${ }^{10} \mathrm{El}$ que hayan sido dispuestos a plena vista descuida el hecho de que los espectadores leen lo que miran no aislando los objetos, sino estableciendo relaciones entre ellos.

Antes de salir de la sala, los transeúntes se enfrenta a un panel explicativo que, mediante texto y un cuadro de doble entrada, ordena la Historia del Arte y la cultura desde el 1300 d. C hasta el 1700 d. C. bajo un criterio historiográfico eurocéntrico, moderno y católico. De más

10 Mediante la conversación con el personal de seguridad de la sala, descubro que se trata de termohigrógrafos, aparatos mecánicos de medición de temperatura y humedad. Miden aproximadamente 30 x $40 \mathrm{~cm}$ cada uno, y si bien controlan los factores atmosféricos de todo el espacio, están ubicados a cada lado del objeto en exhibición, arriba de su base, es decir, compartiendo el recinto demarcador de la obra de arte. Uno de apariencia más discreta fue instalado este mismo año; mide $3 \times 5 \mathrm{~cm}$ aproximadamente, y está ubicado en la parte superior de una de las columnas, fuera de la vista habitual. Sin embargo, los otros dos no fueron removidos. está decir que esta mirada no está señalada como una de las posibles siquiera, con la firma de un investigador, sino grabada como criterio de verdad bajo los títulos "Grandes artistas de los S. XV, XVI y XVII" y "Grandes acontecimientos de los S. XV, XVI y XVII" de forma anónima en acrílico de $20 \mathrm{~mm}$ ocupando $1,5 \mathrm{~m}^{2}$ de pared.

En resumidas cuentas, independientemente del relato curatorial que el Museo quiera proponer, del ordenamiento y formas de disposición de su acervo, la institución no puede incluir en estos programas las obras donadas por la familia Hirsch, siquiera para ponerlas en relación con otras piezas del patrimonio con las que tal vez posibilitarían otras construcciones de sentido. Ambas partes se ven perjudicadas: desde la perspectiva museal, la coherencia del circuito general de la planta baja -independientemente de sus posibles valoraciones - se ve interrumpida por obras, elementos espaciales y formas de distribución que no se corresponden con la linealidad adoptada como criterio general. Por otro lado, las piezas de la colección Hirsch están alejadas de información contextual o de otras obras que les sean afines y permitan descubrir su particularidad estética.

Por ello es que, llegando al final de esta investigación, quisiera puntualizar algunos puntos de cara a pensar el museo como una institución pública que genera sentido sobre el presente, y sobre una de las maneras en que una nación se construye y presenta ante otras, desde su centro político y económico.

En primer lugar, en relación con la cuestión de la identidad nacional presente, que la capital de una nación muestra a sus compatriotas y visitantes extranjeros, quisiera comparar el ya mencionado acervo inaugural del Museo Nacional de Bellas Artes con las obras exhibidas de forma permanente los últimos años.

Como hemos descrito en el primer apartado, según el Primer catálogo de obras del MNBA, en el año 1896 el museo contaba con 144 obras de origen europeo y 14 piezas de origen americano. En el día de hoy ${ }^{11}$ hay exhibidas 379 obras de la primera categoría y 177 De la segunda. Cabe reiterar que 65 de estas últimas, si bien pertenecen a autores de territorio americano, sus escuelas de formación son europeas. Podemos concluir, entonces, que a grandes rasgos el panorama no ha cambiado mucho desde las primeras adquisiciones. Una de las razones es que muchas de las piezas que ocupan

11 Últimos datos obtenidos en mayo de 2018. Ver Tabla 3. 

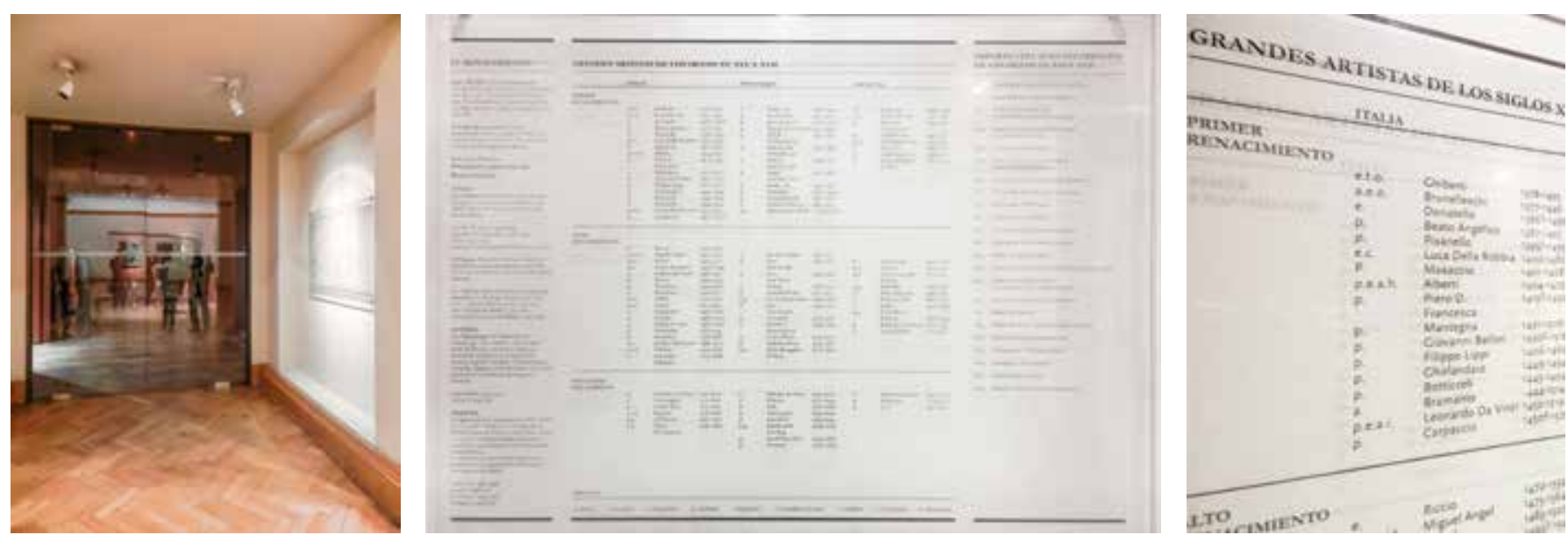

Imagen 4. Cuadro informativo ubicado en la pared posterior este de la sala, anterior a la salida de la exposición.

la categoría más numerosa del acervo ingresaron al patrimonio del museo vía donación.

Es cierto que esta generosidad fue provechosa para aumentar de forma acelerada el acervo en los tiempos inaugurales del museo. Cierto es también que las donaciones no fueron gratuitas, sino que vinieron acompañadas de cargos que hacían de esta nueva propiedad nacional un estatuto compartido de por vida con entidades privadas. Además de la sala que estamos analizando, el caso de la donación Guerrico, por ejemplo, con demanda al museo de por medio, da cuenta de ello. La aceptación de donaciones fue por un lado un modo cómodo de adquisición de piezas, pero por otro, un contrato permanente de lo público con familias particulares que desde el momento en que se vincularon con el museo vienen ejerciendo su poder dentro del espacio sin vistas a ceder. Así, de un modo progresivo, las consecuencias de haber aceptado donaciones en los años 1895, 1906, 1910, 1938, 1942, 1955, 1960, 1963, 1973, 1983, 1986, 1990, se extienden hasta el presente del museo, afectando con su notoria presencia ${ }^{12}$ los planes que pueda tener cada gestión.

El 19 de Julio de 2006, por Resolución SC N.․ 2030/2006, se crea el Comité de Evaluación de Ingreso de Bienes Culturales, con el fin de evaluar los bienes

12 Las obras pertenecientes a donaciones ocupan hoy el $84.05 \%$ de las piezas exhibidas. propuestos para ingresar al patrimonio del MNBA. ${ }^{13} \operatorname{Sin}$ embargo, los cargos de las donaciones son desconocidos por dicho Comité hasta después de haberlas aceptado. ${ }^{14}$ Por otra parte, el Comité de evaluación actúa sobre las propuestas de ingreso posteriores a su fecha de creación, por lo que las colecciones adquiridas hasta esa fecha y sus respectivos cargos continúan vigentes y ordenando la planta baja del museo a la manera arbitraria de la biblioteca china de Borges: arte europeo del siglo XII al XVI, colección Hirsch, Pintura holandesa y flamenca, manierismo y barroco, colección Emilio $P$. Furt, Pintura del Siglo XVIII, etc...

Por lo visto, la puja privada en el seno de la política pública es una característica nacional de la que el campo del arte no está eximido.

13 El 19 de Julio de 2006, por Resolución SC N. 2030/2006, se crea el Comité de Evaluación de Ingreso de Bienes Culturales (art. $1^{\circ}$ ). Según el art. $2^{\circ}$ de dicha Resolución el mismo está integrado por el Director del Museo Histórico Nacional, la Directora del Instituto Nacional de Antropología y Pensamiento Latinoamericano, el Vicepresidente de la Academia Nacional de Bellas Artes y el Director Nacional de Patrimonio y Museos. Y según el Art. 6 de la misma Resolución, en la evaluación de los bienes propuestos para ingresar al patrimonio, “El Comité deberá evaluar la conveniencia de ingreso de bienes al patrimonio de los organismos dependientes de la Dirección Nacional de Patrimonio y Museos, contemplando si el bien responde a la temática del organismo o al carácter de sus colecciones, su valor de mercado, su valoración subjetiva, y la necesidad de contar con dicho bien, ya sea por su condición de objeto único o por la escasez de bienes idénticos o similares".

14 "Se deberá agregar la planilla de cargo patrimonial por triplicado debidamente cumplimentada luego de la intervención del COMITÉ DE EVALUACIÓN DE INGRESO DE BIENES CULTURALES, y siempre que este haya dictaminado favorablemente." (Resolución SC N. 1786/2012, art. 1으, el subrayado es mío). 


\section{Boceto de una síntesis}

Así las cosas, el MNBA podría retratarse en la síntesis que oficia de portada a este trabajo, que resume la inamovible rigidez de la planta baja seguida por los dos pisos superiores, maleables, móviles e incluso desarmables. Mientras que los pisos 1 y 2 reúnen grupos de obras latinoamericanas en escasos períodos de tres meses y relaciones discontinuas entre ellas, la primera planta recibe a los visitantes con la certeza de lo antiguo y europeo, piezas indiscutiblemente artísticas cuyos territorios de origen las respaldan. La sala Hirsch en tanto colección privada de arte europeo pertenece a la planta baja y goza de la estabilidad de este nivel permanente del edificio.

Podemos preguntarnos, entonces, ¿es realmente una donación si la entidad a la que es donada no puede disponer de ella? Los cargos de las colecciones privadas que son introducidas al patrimonio conllevan la imposibilidad de revisionismo por parte del museo. Este se ve obligado a sostener por décadas el mismo modelo inamovible desde 1893. Consecuentemente, la nación reproduce para sus adentros y ante el extranjero una imagen particular que gana fuerza en la repetición: una imagen de miras hacia afuera, con basamento pro Europa y en deshermandad y desconexión tanto con otros países de América - ni decir del resto del globoe incluso con las provincias del interior. El hecho de que las salas permanentes de un museo nacional no puedan ser siquiera reacomodadas pone en duda la capacidad de una nación para repensar su historia desde la contemporaneidad.

En fin, tal vez el sueño modernizador se cumplió eventualmente, dado que uno de sus propósitos era fijar los particulares valores caucásicos como bases universales, y hoy son estos los que representan las salas permanentes del Museo Nacional de un país de colonia. Los argentinos viajamos a Europa y vemos arte europeo en sus museos nacionales. Los europeos viajan a América, y al menos en Buenos Aires, ven lo mismo. Lo propio del territorio en que la institución se inscribe está destinado a las salas temporarias, o incluso a la bodega.

\section{Epílogo}

Esta investigación tuvo lugar durante el año 2018. A principios del 2019, el Museo Nacional empezó un proceso de remodelaciones entre las que debe mencionarse la incorporación de una sala de arte colonial y una de arte precolombino ( $N$. -23 y 24 en el plano, respectivamente). No obstante, tampoco estos cambios significaron una actitud crítica acerca de la perspectiva modernizadora, eurocéntrica y colonial de la institución: las piezas precolombinas exhibidas pertenecientes a un recorte temporal amplio -aproximadamente 1932 años- y a un área geográfica también considerable, alcanzan el número de 310, y no ocupan más que una sala de aproximadamente $10 \times 13 \mathrm{~m}$, mientras que la Colección Hirsch, por ejemplo, exhibe cómodamente 26 obras en un espacio que duplica en superficie a la sala precolonial. Lo que esta diferencia cuantitativa introduce es un problema de tipo museográfico, que ha sido advertido ya repetidamente acerca de los criterios valorativos que subyacen tras la exhibición de arte no europeo bajo la categoría de artesanía.

\section{Referencias}

AAVV (1952). Reportaje a la Pintura argentina de este siglo, Museo Nacional de Bellas Artes, Museos Nacionales y Secretaría de Cultura de la Nación, Archivo general de la Nación, Ministerio de Interior y Transporte. Disponible en: https://vimeo.com/76176492. Consultado en marzo de 2018.

AAVV (1986). Primer Catálogo de las obras del Museo de Bellas Artes, Buenos Aires, Museo Nacional de Bellas Artes.

(2006). Guía MNBA, Buenos Aires, Asociación de Amigos del Museo Nacional de Bellas Artes.

(2006). Resolución SC N. 2030/2006, en Normativa, Servicio Nacional de Inventarios de Patrimonio, formato electrónico: https://senip.cultura. gob.ar/normativa/ Fecha de visita: 13/06/18.

. (2007). Guía, Buenos Aires, Asociación de Amigos del Museo Nacional de Bellas Artes.

(2009). Guía del MNBA Buenos Aires, Asociación de Amigos del Museo Nacional de Bellas Artes.

(2012). Resolución SC N. 1786/12, en

Normativa, Servicio Nacional de Inventarios de Patrimonio, formato electrónico: https://senip.cultura. gob.ar/normativa/ Fecha de visita: 13/06/18. 
. (2015). Guía del MNBA Buenos Aires,

Asociación de Amigos del Museo Nacional de Bellas Artes.

Alloway, L. (1969). The Venice Biennale. 1895-1968 from salon to goldfish bowl. Londres: Faber and Faber.

Amigo, R. (2010). “Presentación”, en Museo Nacional de Bellas Artes. Colección, Volumen 1, p. 17. Buenos Aires: Asociación de Amigos del Museo Nacional de Bellas Artes.

Bal, M. (2010). "Arte para lo político", en: Revista de Estudios Visuales, N.7, pp. 40-65.
Cerón, J. (2011). El museo como representación de los conflictos culturales. Calle 14 Revista De investigación En El Campo Del Arte, 5(7), 142-153. https://doi. org/10.14483/udistrital.jour.c14.2011.2.a11

Herrera, M. J. (2010). “El Museo Nacional de Bellas Artes. Historia, gestiones y curaduría", en Museo Nacional de Bellas Artes. Colección, Volumen 1, pp. 18-35. Buenos Aires: Asociación de Amigos del Museo Nacional de Bellas Artes.

Wechsler, D. (2012). Pensar con imágenes. Un ejercicio curatorial con la Colección del Banco Ciudad. Buenos Aires: Fundación OSDE. 